\title{
Differential expression of PD-L1 and PD-L2 is associated with the tumor microenvironment of TILs and M2 TAMs and tumor differentiation in non-small cell lung cancer
}

\author{
RYOTA SUMITOMO ${ }^{1-3}$, CHENG-LONG HUANG ${ }^{1,2}$, MASAAKI FUJITA ${ }^{2}$, \\ HIROYUKI $\mathrm{CHO}^{1}$ and HIROSHI DATE ${ }^{3}$
}

\begin{abstract}
Departments of ${ }^{1}$ Thoracic Surgery and ${ }^{2}$ Oncology, Tazuke Kofukai Medical Research Institute, Kitano Hospital, Kita-ku, Osaka 530-8480; ${ }^{3}$ Department of Thoracic Surgery, Faculty of Medicine, Kyoto University, Shogoin, Sakyo-ku, Kyoto 606-8507, Japan
\end{abstract}

Received October 6, 2021; Accepted February 1, 2022

DOI: 10.3892/or.2022.8284

\begin{abstract}
To improve the treatment strategy of immune-checkpoint inhibitors for non-small cell lung cancer (NSCLC), a comprehensive analysis of programmed death-ligand (PD-L)1 and PD-L2 expression is clinically important. The expression of PD-L1 and PD-L2 on both tumor cells (TCs) and tumor-infiltrating immune cells (ICs) was investigated, with respect to tumor-infiltrating lymphocytes (TILs) and M2 tumor-associated macrophages (TAMs), which are key components of the tumor microenvironment, in 175 patients with resected NSCLC. The TIL and M2 TAM densities were associated with the expression of PD-L1 on the two TCs (both $\mathrm{P}<0.0001)$ and ICs (both $\mathrm{P}<0.0001)$. The TIL and M2 TAM densities were also associated with the expression of PD-L2 on both TCs ( $\mathrm{P}=0.0494$ and $\mathrm{P}=0.0452$, respectively) and ICs $(\mathrm{P}=0.0048$ and $\mathrm{P}=0.0125$, respectively). However, there was no correlation between the percentage of PD-L1-positive TCs and the percentage of PD-L2-positive TCs ( $\mathrm{r}=0.019 ; \mathrm{P}=0.8049)$. Meanwhile, tumor differentiation was significantly associated with the PD-L1 expression on TCs and ICs ( $\mathrm{P}=0.0002$ and $\mathrm{P}<0.0001$, respectively). By contrast, tumor differentiation was inversely associated with the PD-L2 expression on both TCs and ICs $(\mathrm{P}=0.0260$ and $\mathrm{P}=0.0326$, respectively). In conclusion, the combined evaluation of PD-L1 and PD-L2 expression could be clinically important in the treatment strategy of immune-checkpoint inhibitors in patients with NSCLC. In
\end{abstract}

Correspondence to: Dr Cheng-Long Huang, Department of Thoracic Surgery, Tazuke Kofukai Medical Research Institute, Kitano Hospital, 2-4-20 Ohgimachi, Kita-ku, Osaka 530-8480, Japan

E-mail: chuang@kitano-hp.or.jp

Key words: programmed death-ligand 1/2, tumor cell, immune cell, macrophage, lymphocyte, tumor microenvironment, differentiation, lung cancer particular, the evaluation of PD-L2 expression may be necessary for patients with PD-L1-negative NSCLC.

\section{Introduction}

Non-small cell lung cancer (NSCLC), accounting for $\sim 85 \%$ of all cases of lung cancer, remains to be the leading cause of cancer-related mortality worldwide, despite the availability of advanced cytotoxic chemotherapies and molecular-targeted therapies, such as EGFR-tyrosine kinase inhibitors $(1,2)$. However, recently, agents that target the programmed death-1 (PD-1)/programmed death-ligand 1 (PD-L1) axis, such as immune-checkpoint inhibitors, have been widely used as a standard treatment for patients with metastatic NSCLC (3-5). Pembrolizumab, an anti-PD-1 antibody, has been approved as monotherapy in patients with tumors that have highly upregulated expression of PD-L1 on tumor cells (TCs) (4). This finding made PD-L1 testing a mandatory diagnostic test during treatment planning in patients with NSCLC. Furthermore, effective clinical response to atezolizumab, an anti-PD-L1 antibody, is observed not only in patients with tumors with high PD-L1 expression on TCs, but also in patients with tumors that expressed high levels of PD-L1 on tumor-infiltrating immune cells (ICs) (6). These observations suggest that the PD-L1 expression not only on TCs but also ICs serves an important role in regulating the anti-tumor $\mathrm{T}$ cell response. In addition, the PD-L1 expression on TCs and ICs is reported to be affected by microenvironment stimuli, including tumor-infiltrating lymphocytes (TILs) and M2 tumor-associated macrophages (TAMs) $(7,8)$.

On the other hand, recent clinical studies report that PD-L2, another PD-1 ligand, is also widely expressed in numerous types of cancer, including NSCLC (9-14). Several studies reveal that PD-L2 is also expressed by both TCs and various ICs, depending on the microenvironment stimuli (15-17). In addition, experimental studies report that PD-L2-expressing TCs are resistant to treatment with anti-PD-L1 antibody alone and that this resistance is overcome by an anti-PD-1 antibody or in combination with an anti-PD-L2 antibody $(18,19)$. A clinical study reports that 
clinical response to pembrolizumab in patients with head and neck squamous cell carcinoma may be related partly to blockade of PD-1/PD-L2 interactions (20). However, the clinical significance of the PD-L2 expression in NSCLC is still controversial (7,9-11).

Taken together, to improve the treatment strategy of immune-checkpoint inhibitors for patients with NSCLC, a comprehensive analysis of the biological mechanisms and clinical significance of PD-L1 and PD-L2 expression was considered to be clinically important. Therefore, a the present study was performed to evaluate the expression of PD-L1 and PD-L2 on both TCs and ICs in patients with NSCLC. In addition, the association between TILs and M2 TAMs, which are key components of the tumor microenvironment (TME), on the expression of PD-L1 and PD-L2 was also analyzed.

\section{Materials and methods}

Patients. Consecutive 175 patients with NSCLC, who underwent surgery at the Department of Thoracic Surgery, Kitano Hospital (Osaka, Japan) between November 2011 and December 2014, were included. The present study was approved by the Ethics Committee (approval no. P181200300) and written informed consent was provided from each patient. Pathological staging was determined using the 8th Tumor Node Metastasis (TNM) classification system (21). The histological type and the grade of differentiation of the tumors were determined according to the classification system developed by the World Health Organization (22). The medical records and histopathological diagnosis from the patients were fully documented.

Immunohistochemistry. Immunohistochemical studies were performed to evaluate the TIL distribution by CD3 staining, the M2 TAM distribution by CD163 staining (8,23), PD-L1 expression on TCs and ICs by the Ventana SP263 assay and PD-L2 expression on TCs and ICs, using the Ventana BenchMark GX system (Ventana Medical Systems; Roche Diagnostics), according to the recommended protocol. The following antibodies were used: Rabbit monoclonal anti-human CD3 (clone 2GV6; prediluted; Ventana Medical Systems; Roche Diagnostics), PD-L1 (clone SP263; prediluted; Ventana Medical Systems; Roche Diagnostics) (24) and PD-L2 (cat. no. 18251-1-AP; 1:200; ProteinTech Group, Inc.) and mouse monoclonal anti-human CD163 (clone 760-4437; prediluted; Ventana Medical Systems; Roche Diagnostics). The tissues were fixed in 10\% neutral-buffered formalin for $24 \mathrm{~h}$ at room temperature. After dehydration in graded ethanol series followed by xylene at room temperature, the tissues were embedded in paraffin at $60^{\circ} \mathrm{C}$. Formalin-fixed paraffin-embedded tissue was cut into $4-\mu \mathrm{m}$ sections and mounted on poly-L-lysine-coated slides. The sections were deparaffinized and rehydrated using EZ Prep (Ventana Medical Systems; Roche Diagnostics) at $75^{\circ} \mathrm{C}$. Antigen retrieval was performed using Cell Conditioner 1 (Ventana Medical Systems; Roche Diagnostics) for $64 \mathrm{~min}$ at $100^{\circ} \mathrm{C}$ against CD3, PD-L1 and PD-L2 and $32 \mathrm{~min}$ at $100^{\circ} \mathrm{C}$ against CD163. The sections were then incubated with the specific primary antibody for $16 \mathrm{~min}$ at $37^{\circ} \mathrm{C}$ against $\mathrm{CD} 3, \mathrm{CD} 163$ and PD-L1 and $2 \mathrm{~h}$ at $37^{\circ} \mathrm{C}$ for PD-L2. Subsequently, the sections were treated with the OptiView HQ Linker (Ventana Medical Systems; Roche Diagnostics) for $8 \mathrm{~min}$ at $37^{\circ} \mathrm{C}$ and the OptiView HRP Multimer (Ventana Medical Systems; Roche Diagnostics) for $8 \mathrm{~min}$ at $37^{\circ} \mathrm{C}$. Finally, counterstaining was performed with Mayer's hematoxylin and Scott's tap water bluing reagent at $37^{\circ} \mathrm{C}$.

The evaluation of the stained tissue sections was performed by two investigators (RS and CLH) blinded to the study. The cases with discrepancies were jointly re-evaluated until a consensus was reached. For CD3 and CD163 staining, the five most representative high-power fields (magnification, $\mathrm{x} 400$; $0.0625 \mathrm{~mm}^{2}$ ) of the tumor stroma were selected. Tumor stroma was defined as the area where tumor stromal cells accounted for $>70 \%$ of the total cells (25). The number of CD3-positive cells and CD163-positive cells in each area was counted and the average number of fields in each area was calculated. Finally, the CD3-positive cell density in the tumor stroma (TIL density) and the CD163-positive macrophage density in the tumor stroma (M2 TAM density) were defined as the cell number per $\mathrm{mm}^{2}$. PD-L1 and PD-L2 expression was calculated as the percentage of membrane staining on TCs or ICs, respectively, in the overall area of the tumor, regardless of intensity.

Statistical analysis. The statistical significances regarding continuous variables were assessed using either a t-test, ANOVA with Bonferroni/Dunn post hoc test or Pearson's correlation coefficient. Categorical variables were compared using a $\chi^{2}$ test. Statistical analyses were performed using SPSS v23.0 for Windows (IBM Corp.). All P-values were based on the two-sided statistical analysis and $\mathrm{P}<0.05$ was considered to indicate a statistically significant difference.

\section{Results}

Distribution and clinical significance of TILs among resected NSCLCs. Immunohistochemistry for CD3 exhibited a membranous and cytoplasmic staining pattern (Fig. 1A, C and E). The TIL density varied among the 175 tumor tissues (mean \pm standard deviation, $948.1 \pm 890.6$; Table I). As the TIL density cut-off (524) demonstrated the highest significance with respect to the percentage of PD-L1-positive TCs and PD-L2-positive TCs, the sample was classified as TIL-high when the TIL density was $>524$. A total of 71 tumors $(40.6 \%)$ were classified as TIL-low and 104 tumors (59.4\%) were classified as TIL-high. With respect to tumor histology, the TIL density was significantly higher in squamous cell carcinoma compared with that in adenocarcinoma $(\mathrm{P}=0.0206)$. In addition, with respect to tumor differentiation, the TIL density was significantly higher in moderately and poorly differentiated tumors compared with that in well-differentiated tumors $(\mathrm{P}=0.0130)$.

Distribution and clinical significance of M2 TAMs among resected NSCLCs. Immunohistochemistry for CD163 exhibited a membranous and cytoplasmic staining pattern (Fig. 1G). The M2 TAM density also varied among the 175 tumor tissues (mean \pm standard deviation, 382.5 \pm 381.9 ; Table I). There was a weak correlation between TIL and M2 TAM densities $(\mathrm{r}=0.262 ; \mathrm{P}=0.0004 ;$ Fig. 2$)$. The sample was classified as $\mathrm{M} 2$ TAM-high when the M2 TAM density was $>380$ due to the highest significance in the level of $\mathrm{C}$-reactive protein, a marker 


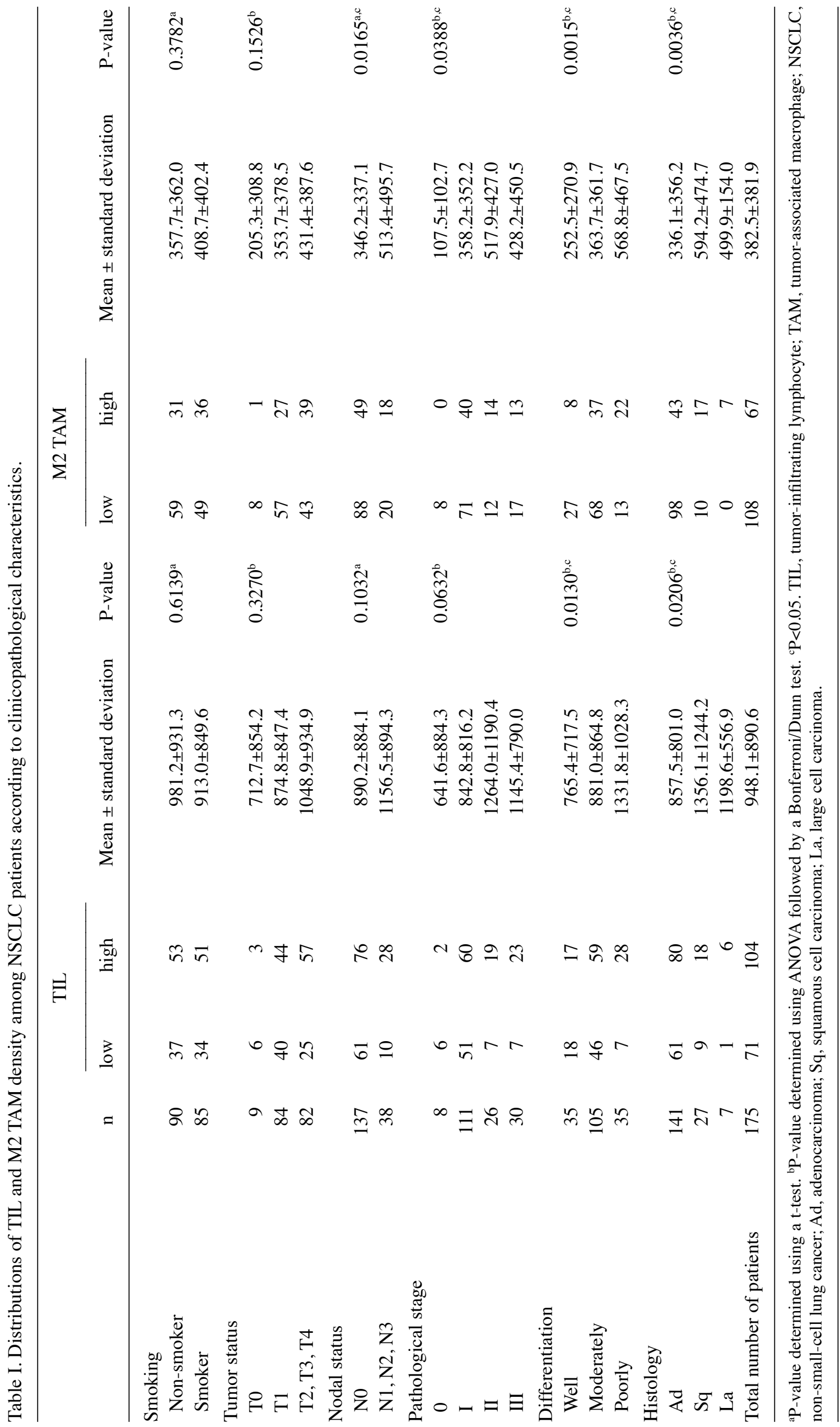




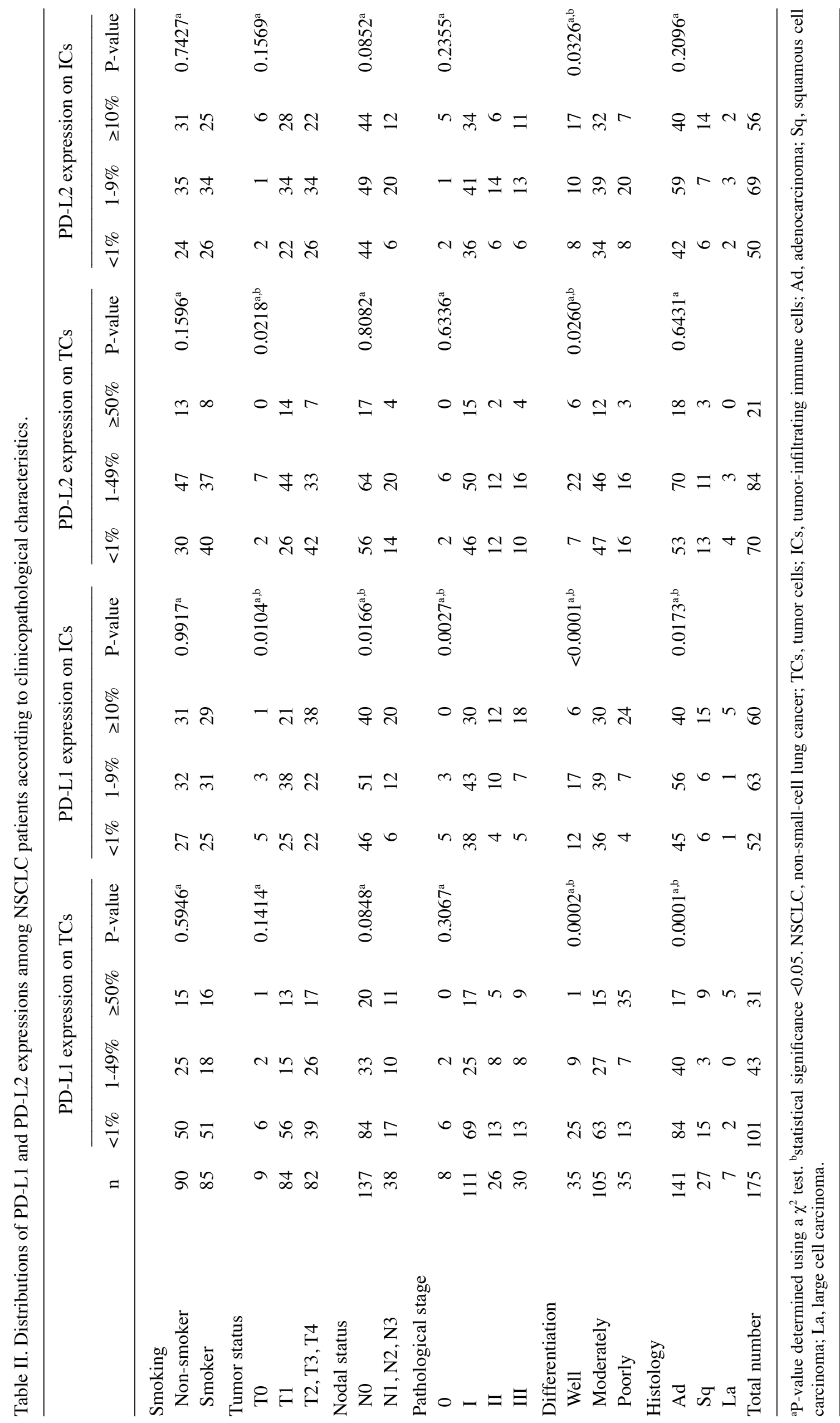



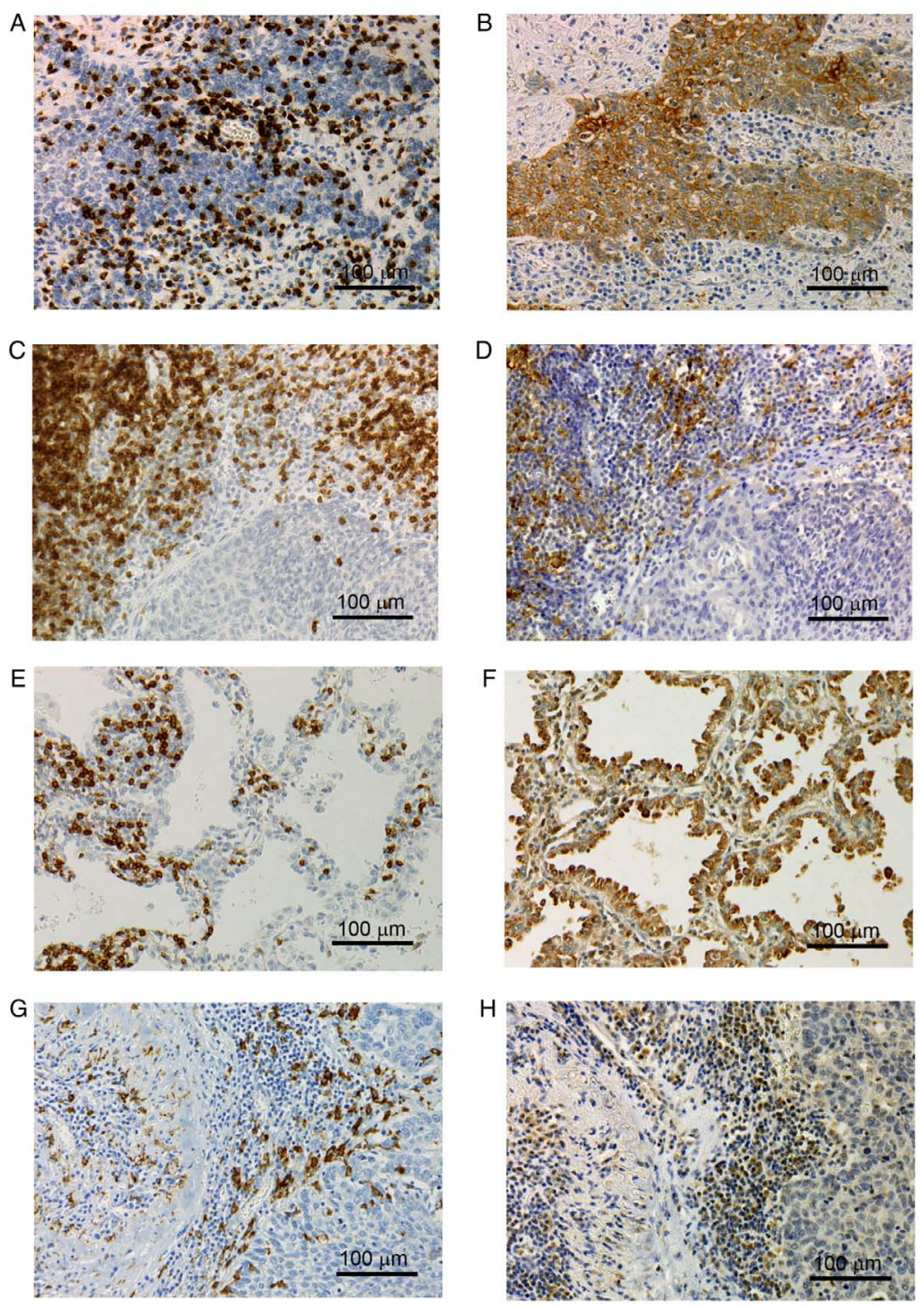

Figure 1. Immunostaining of lung cancer. (A) A squamous cell carcinoma with a high density of TIL and (B) positive expression of PD-L1 on TCs. (C) A squamous cell carcinoma with a high density of TIL and (D) positive expression of PD-L1 on ICs. (E) An adenocarcinoma with a high density of TIL and (F) positive expression of PD-L2 on TCs. (G) A squamous cell carcinoma with a high density of M2 TAM and (H) positive expression of PD-L2 on ICs. Scale bar $=100 \mu \mathrm{m}$. TIL, tumor-infiltrating lymphocyte; PD-L, programmed death-ligand; TCs, tumor cells; ICs, tumor-infiltrating immune cells; TAM, tumor-associated macrophage.

of the inflammatory response, as previously reported (23). A total of 108 tumors $(61.7 \%)$ were classified as M2 TAM-low and 67 tumors $(38.3 \%)$ were classified as M2 TAM-high. The M2 TAM density was also significantly higher in squamous cell carcinoma compared with that in adenocarcinoma $(\mathrm{P}=0.0036)$. The M2 TAM density was also significantly higher in poorly differentiated tumors compared with that in well- and moderately differentiated tumors $(\mathrm{P}=0.0015)$. Furthermore, the M2 TAM density was significantly higher in node-positive tumors and advanced stage $(\mathrm{P}=0.0165$ and $\mathrm{P}=0.0388$, respectively).

Expression of PD-L1 on TCs and ICs with respect to TILs and M2 TAMs. The percentage of PD-L1-positive TCs varied among the 175 tumor tissues (mean \pm standard deviation; 15.6 $\pm 27.0 \%$; Fig. 1B). PD-L1 expression on TCs was significantly higher in squamous cell carcinoma compared with that in adenocarcinoma $(\mathrm{P}=0.0001)$.

The percentage of PD-L1-positive ICs also varied (mean \pm standard deviation, 9.4 $\pm 10.9 \%$; Fig. 1D). PD-L1

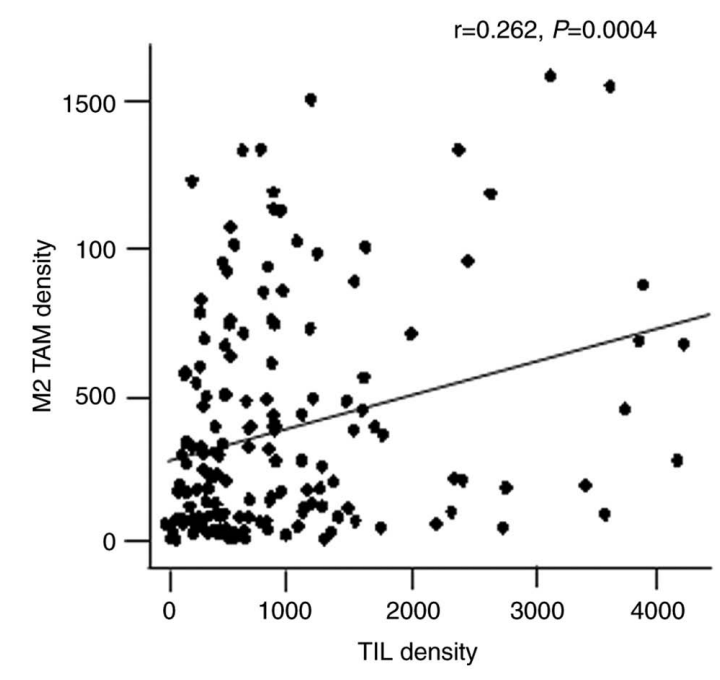

Figure 2. Association between TIL and M2 TAM densities. TIL, tumor-infiltrating lymphocyte; TAM, tumor-associated macrophage. TIL, tumor-infiltrating lymphocyte; TAM, tumor-associated macrophage. 

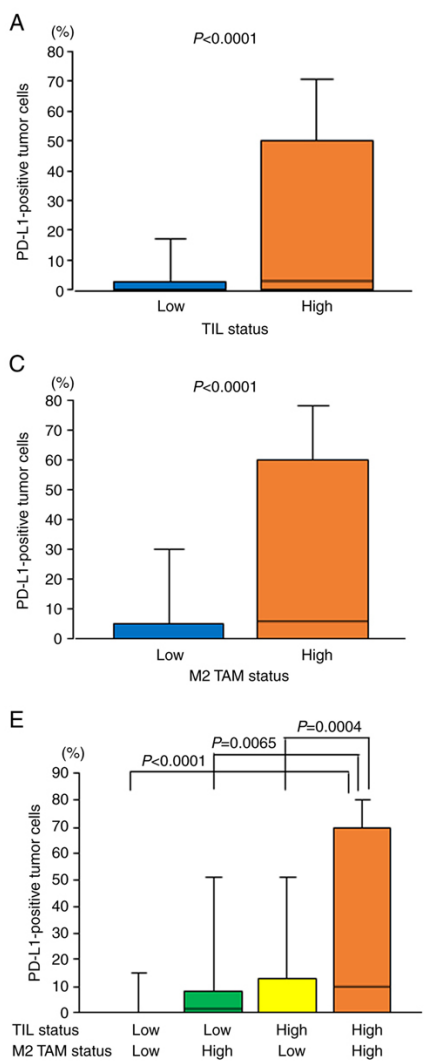
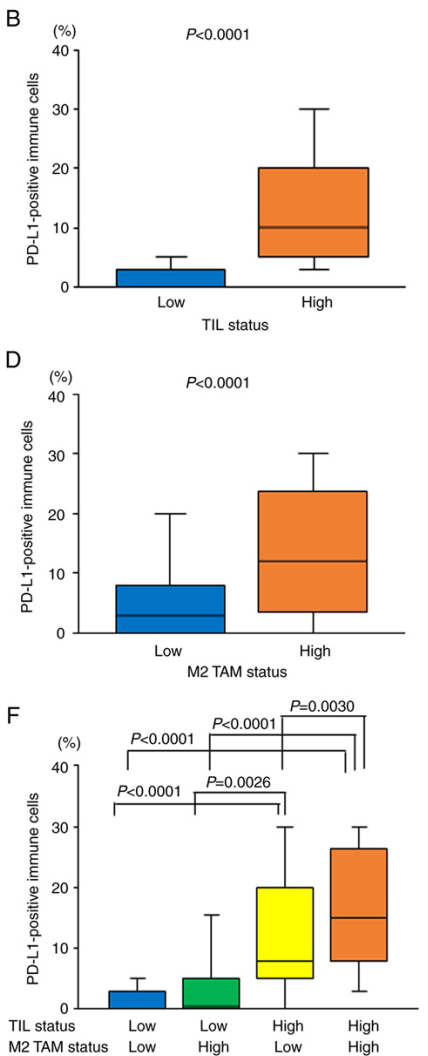
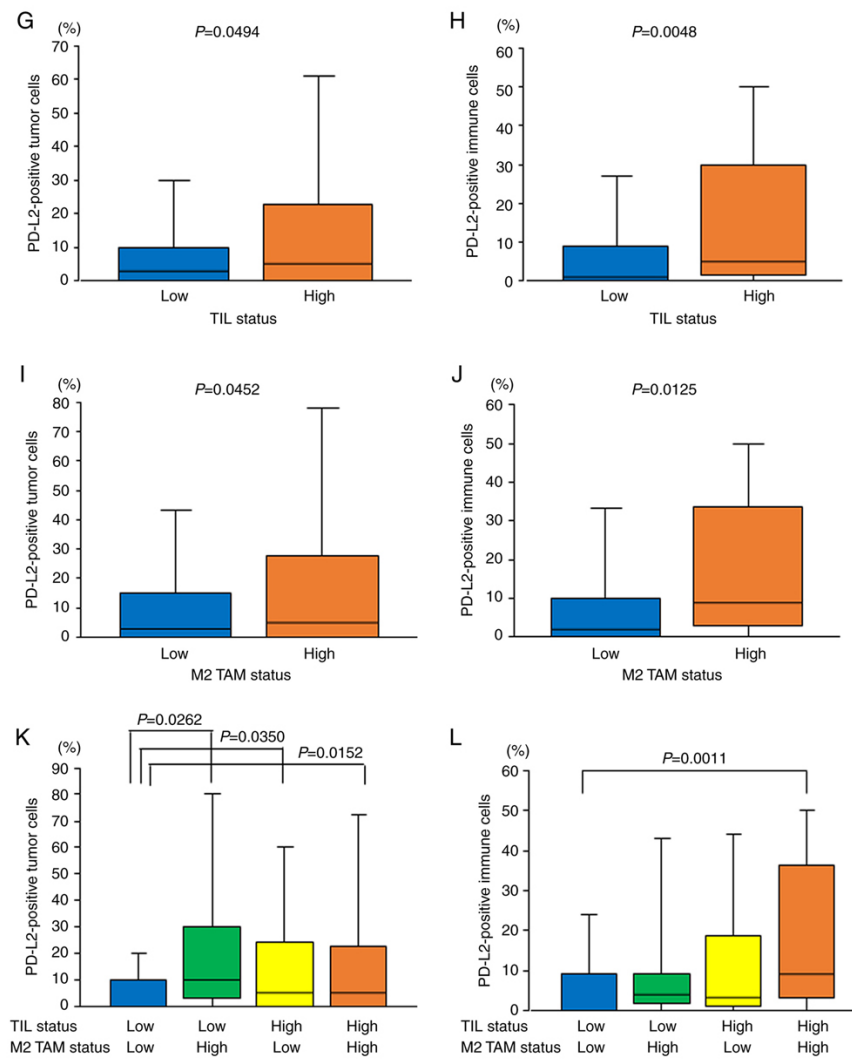

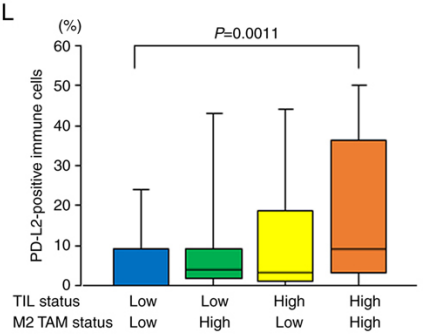

Figure 3. PD-L1 expression on (A) TCs and (B) ICs with respect to TIL density. PD-L1 expression on (C) TCs and (D) ICs with respect to M2 TAM density. PD-L1 expression on (E) TCs and (F) ICs with respect to TIL and M2 TAM densities. PD-L2 expression on (G) TCs and (H) ICs with respect to TIL density. PD-L2 expression on (I) TCs and (J) ICs with respect to M2 TAM density. PD-L2 expression on (K) TCs and (L) ICs with respect to TIL and M2 TAM densities. PD-L, programmed death-ligand; TCs, tumor cells; ICs, tumor-infiltrating immune cells; TAM, tumor-associated macrophage; TIL, tumor-infiltrating lymphocyte.

expression on ICs was also significantly higher in squamous cell carcinoma compared with that in adenocarcinoma $(\mathrm{P}=0.0173)$. Furthermore, PD-L1 expression on ICs was significantly associated with tumor status, nodal status and pathological stage ( $\mathrm{P}=0.0104, \mathrm{P}=0.0166$ and $\mathrm{P}=0.0027$, respectively).

With respect to TILs, the TIL density was significantly correlated with the percentage of PD-L1-positive TCs $(\mathrm{r}=0.365 ; \mathrm{P}<0.001)$. The percentage of PD-L1-positive TCs was significantly higher in the TIL-high group compared with that in the TIL-low group $(22.2 \pm 30.8 \%$ vs. $6.1 \pm 16.0 \%$; $\mathrm{P}<0.0001$; Fig. 3A). Furthermore, the TIL density was also significantly correlated with the percentage of PD-L1-positive ICs ( $\mathrm{r}=0.751$; $\mathrm{P}<0.001)$. The percentage of PD-L1-positive ICs was significantly higher in the TIL-high group compared with that in the TIL-low group (14.4 $\pm 11.2 \%$ vs. $1.9 \pm 3.5 \%$; $\mathrm{P}<0.0001$; Fig. 3B).

As previously reported (8), with respect to M2 TAMs, the M2 TAM density was significantly correlated with the percentage of PD-L1-positive TCs $(r=0.389$; $\mathrm{P}<0.001)$. The percentage of PD-L1-positive TCs was significantly higher in the M2 TAM-high group compared with that in the M2 TAM-low group $(26.5 \pm 32.3 \%$ vs. $8.9 \pm 20.5 \%$; $\mathrm{P}<0.0001$; Fig. 3C). Furthermore, the M2 TAM density was significantly correlated with the percentage of PD-L1-positive ICs $(r=0.375$; $\mathrm{P}<0.001)$. The percentage of PD-L1-positive ICs was significantly higher in the M2 TAM-high group compared with that in the M2 TAM-low group $(14.2 \pm 12.0 \%$ vs. $6.4 \pm 8.8 \%$; $\mathrm{P}<0.0001$; Fig. 3D).
With respect to the combined evaluation of TILs and M2 TAMs, the percentage of PD-L1-positive TCs was significantly the highest in both the TIL-high and M2 TAM-high tumors (Fig. 3E). The percentage of PD-L1-positive ICs was also significantly the highest in both the TIL-high and M2 TAM-high tumors (Fig. 3F).

Expression of PD-L2 on TCs and ICs with respect to TILs and M2 TAMs. The percentage of PD-L2-positive TCs varied among the 175 tumor tissues (mean \pm standard deviation, $14.6 \pm 22.9 \%$; Fig. $1 \mathrm{~F}$ ) and there were $<1 \%$ in $70(40.0 \%)$ tumors, $1-49 \%$ in $84(48.0 \%)$ tumors and $\geq 50 \%$ in $21(12.0 \%)$ tumors (Table II). The percentage of PD-L2-positive ICs also varied (mean \pm standard deviation, $12.5 \pm 18.4 \%$; Fig. $1 \mathrm{H}$ ) and there were $<1 \%$ in $50(28.6 \%)$ tumors, $1-9 \%$ in $69(39.4 \%)$ tumors and $\geq 10 \%$ in $56(32.0 \%)$ tumors (Table II).

With respect to TILs, the percentage of PD-L2-positive TCs was significantly higher in the TIL-high group compared with that in the TIL-low group $(17.4 \pm 25.9 \%$ vs. $10.5 \pm 17.0 \%$; $\mathrm{P}=0.0494$; Fig. 3G). In addition, the TIL density was significantly correlated with the percentage of PD-L2-positive ICs $(\mathrm{r}=0.226 ; \mathrm{P}=0.003)$. The percentage of PD-L2-positive ICs was significantly higher in the TIL-high group compared with that in the TIL-low group $(15.7 \pm 19.7 \%$ vs. $7.8 \pm 15.3 \%$; $\mathrm{P}=0.0048$; Fig. 3H).

With respect to M2 TAMs, the percentage of PD-L2-positive TCs was significantly higher in the M2 
TAM-high group compared with that in the M2 TAM-low group $(19.0 \pm 27.9 \%$ vs. $11.9 \pm 18.9 \%$; $\mathrm{P}=0.0452$; Fig. $3 \mathrm{I})$. Furthermore, the percentage of PD-L2-positive ICs was also significantly higher in the M2 TAM-high group compared with that in the M2 TAM-low group $(16.9 \pm 19.8 \%$ vs. $9.8 \pm 17.0 \%$; $\mathrm{P}=0.0125$; Fig. 3J).

With respect to the combined evaluation of TILs and M2 TAMs, the percentage of PD-L2-positive TCs was significantly the lowest in both the TIL-low and M2 TAM-low tumors (Fig. 3K). The percentage of PD-L2-positive ICs was significantly lower in both the TIL-low and M2 TAM-low tumors compared with that in both the TIL-high and M2 TAM-high tumors $(\mathrm{P}=0.0011$; Fig. 3L).

Correlations between the expression of $P D-L 1$ and $P D-L 2$ on the TCs and ICs among resected NSCLC. There was no correlation between the percentage of PD-L1-positive TCs and the percentage of PD-L2-positive TCs $(r=0.019$; $\mathrm{P}=0.8049$; Fig. 4A). On the other hand, the percentage of PD-L1-positive TCs was significantly correlated with the percentage of PD-L1-positive ICs ( $\mathrm{r}=0.396$; $\mathrm{P}<0.0001$; Fig. 4B). In addition, the percentage of PD-L2-positive TCs also was significantly correlated with the percentage of PD-L2-positive ICs ( $\mathrm{r}=0.488$; $\mathrm{P}<0.0001$; Fig. 4C).

Expression of $P D-L 1$ and $P D-L 2$ with respect to tumor differentiation. PD-L1 expression on TCs was significantly associated with tumor differentiation $(\mathrm{P}=0.0002$; Table II), as previously reported (8). The percentage of PD-L1-positive TCs was $6.0 \pm 17.0 \%$ in well-differentiated tumors, $13.1 \pm 24.2 \%$ in moderately differentiated tumors and $32.8 \pm 34.9 \%$ in poorly differentiated tumors. The percentage of PD-L1-positive TCs was significantly higher in poorly differentiated tumors compared with that in well- and moderately differentiated tumors $(\mathrm{P}<0.0001$ and $\mathrm{P}=0.0001$, respectively; Fig. 5A).

Furthermore, PD-L1 expression on ICs was also significantly associated with tumor differentiation $(\mathrm{P}<0.0001$; Table II), as previously reported (8). The percentage of PD-L1-positive ICs was $5.7 \pm 8.6 \%$ in well-differentiated tumors, $8.3 \pm 10.0 \%$ in moderately differentiated tumors and $16.2 \pm 12.6 \%$ in poorly differentiated tumors. The percentage of PD-L1-positive ICs was significantly higher in poorly differentiated tumors compared with that in well- and moderately differentiated tumors $(\mathrm{P}<0.0001$ and $\mathrm{P}=0.0001$, respectively; Fig. 5B).

On the other hand, PD-L2 expression on TCs was inversely associated with tumor differentiation ( $\mathrm{P}=0.0260$; Table II). The percentage of PD-L2-positive TCs was $23.5 \pm 25.9 \%$ in well-differentiated tumors, $13.4 \pm 22.4 \%$ in moderately differentiated tumors and $9.2 \pm 19.1 \%$ in poorly differentiated tumors. The percentage of PD-L2-positive TCs was significantly higher in well-differentiated tumors compared with that in poorly and moderately differentiated tumors $(\mathrm{P}=0.0088$ and $\mathrm{P}=0.0234$, respectively; Fig. 5C).

The PD-L2 expression on ICs was also inversely associated with tumor differentiation ( $\mathrm{P}=0.0326$; Table II). The percentage of PD-L2-positive ICs was $19.3 \pm 22.5 \%$ in well-differentiated tumors, $11.4 \pm 17.4 \%$ in moderately differentiated tumors and $9.1 \pm 15.3 \%$ in poorly differentiated tumors. The percentage of
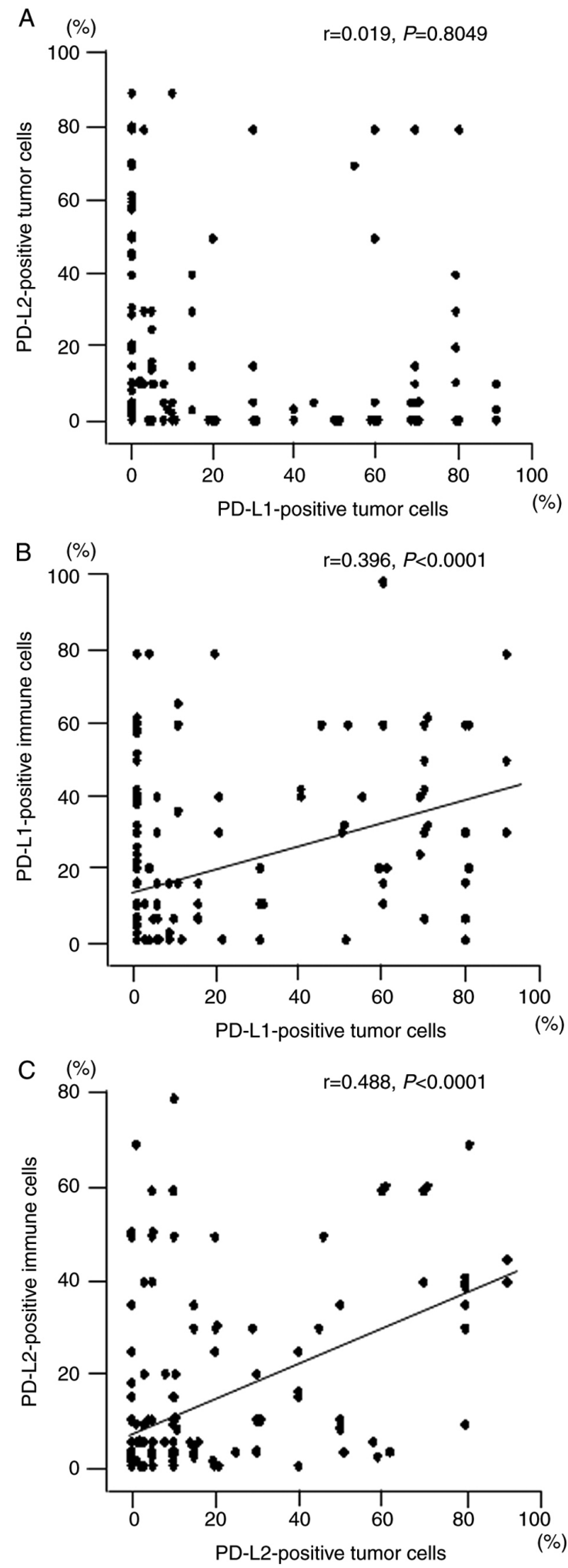

Figure 4. (A) Correlation between PD-L1 expression on TCs and PD-L2 expression on TCs. (B) Correlation between PD-L1 expression on TCs and PD-L1 expression on ICs. (C) Correlation between PD-L2 expression on TCs and PD-L2 expression on ICs. PD-L, programmed death-ligand; TCs, tumor cells; ICs, tumor-infiltrating immune cells.

PD-L2-positive ICs was significantly higher in well-differentiated tumors compared with that in poorly and moderately differentiated tumors $(\mathrm{P}=0.0196$ and $\mathrm{P}=0.0269$, respectively; Fig. 5D). 
A

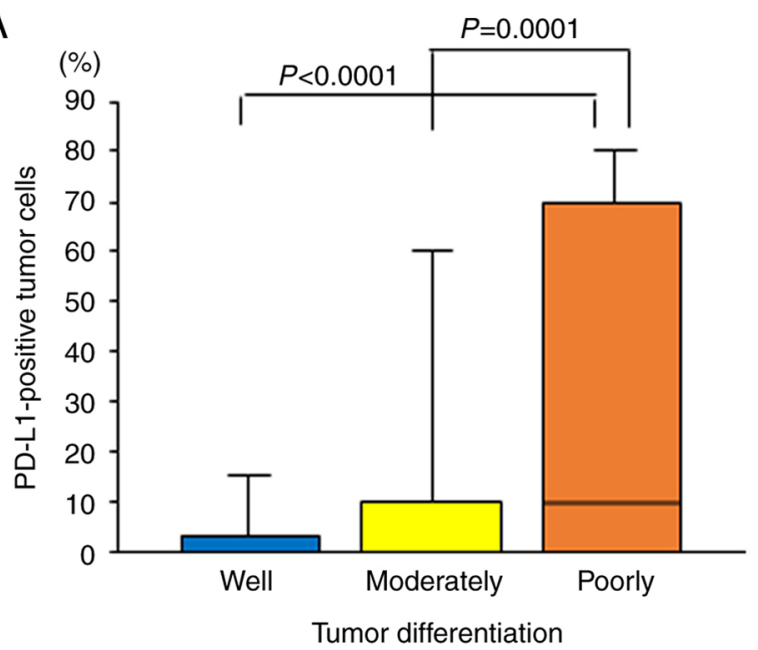

C

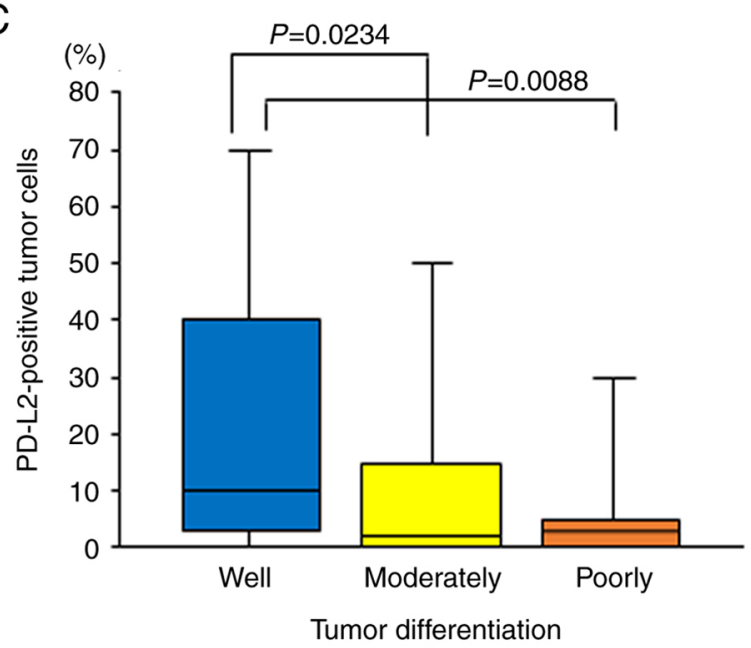

B

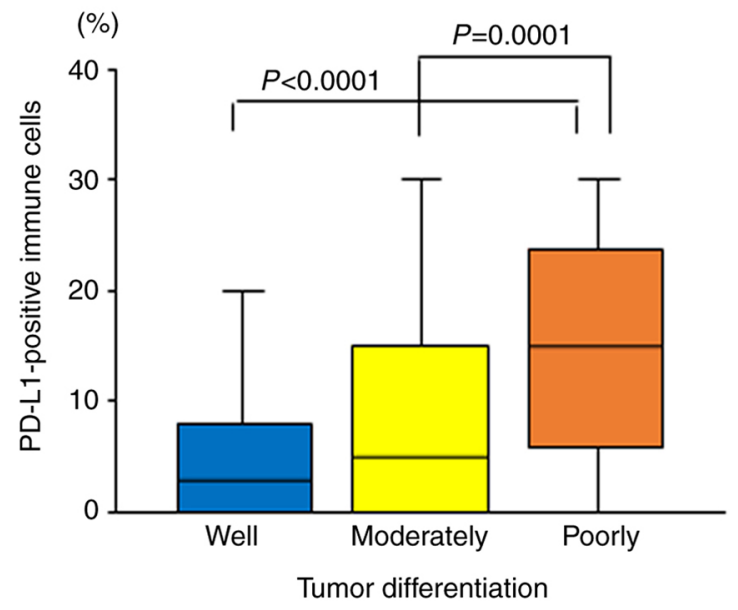

D

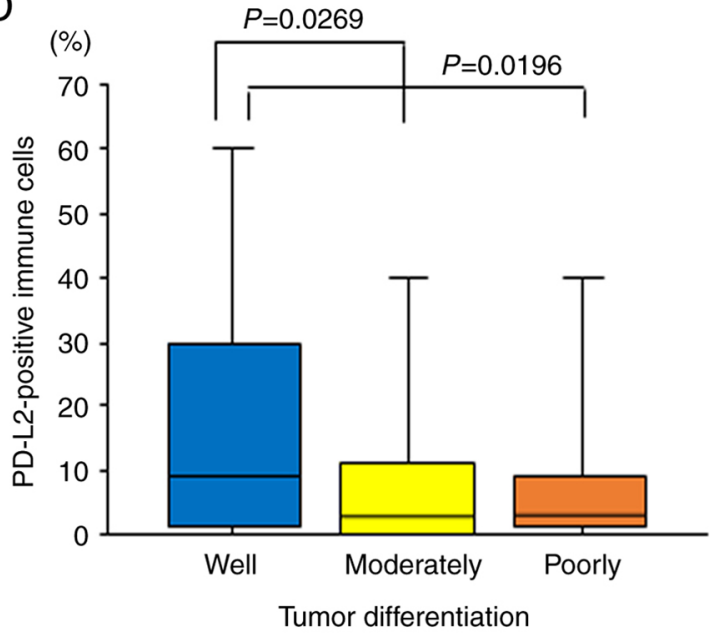

Figure 5. PD-L1 expression on (A) TCs and (B) ICs with respect to tumor differentiation. PD-L2 expression on (C) TCs and (D) ICs with respect to tumor differentiation. TCs, tumor cells; ICs, tumor-infiltrating immune cells. PD-L, programmed death-ligand; TCs, tumor cells; ICs, tumor-infiltrating immune cells.

\section{Discussion}

A comprehensive study on PD-L1 and PD-L2 expression on both TCs and ICs in NSCLC was performed. A recent study reports that $\mathrm{PD}-\mathrm{L} 1$ has predominant roles in Th1-type immunity whereas PD-L2 is involved in Th2-type immunity (26). In addition, to elucidate the biological mechanisms of their regulation, TILs and M2 TAMs, which are key components of the TME and associated with tumor progression, were investigated. The evaluation of PD-L1 and PD-L2 on both TCs and ICs is clinically important and immunohistochemistry is an appropriate method for the design of the present study. A previous study reports that the Ventana PD-L1 (SP-263) assay is clinically useful for PD-L1 staining on both TCs and ICs (24). In addition, the PD-L2 expression using the Ventana system also exhibited a clear staining on both TCs and ICs in the present study.

Consequently, the present study revealed that the TIL density was strongly associated with the PD-L1 expression on both TCs and ICs. On the other hand, PD-L2 was widely expressed not only on TCs, but also on ICs in NSCLC. In addition, the TIL density was also associated with PD-L2 expression on both
TCs and ICs. Initially, $\mathrm{CD}^{+}$or $\mathrm{CD}^{+}{ }^{+} \mathrm{T}$ cells and NK cells are known to induce PD-L1 expression by producing interferon (IFN)- $\gamma(27,28)$. TILs have been reported to be an important cause of PD-L1 expression on ICs, such as lymphatic endothelial cells, macrophages and monocytes (29-31). Numerous clinical studies have also revealed that TILs are associated with PD-L1 expression in human cancer, including NSCLC $(7,32,33)$. In addition, previous studies report that TILs are also associated with PD-L2 expression in human cancer $(33,34)$.

Based on the physiological or pathological situation, macrophages can be polarized into various phenotypes with different biological properties, such as tumor-inhibiting M1 macrophages and tumor-promoting M2 macrophages $(35,36)$. During tumor progression, Th2-derived cytokines originating from TCs and stromal cells can induce the production of M2 TAMs in the TME, which can promote tumor cell proliferation (37). In fact, the M2 TAM density was associated with nodal status and pathological stage in the present study. Thus, M2 TAM-high tumors have more aggressive potential in NSCLC (23).

On the other hand, our previous study found that the M2 TAM density was strongly associated with PD-L1 expression 
on both TCs and ICs (8). In addition, the present study demonstrated that the M2 TAM density was also associated with the PD-L2 expression on both TCs and ICs. Experimental studies report that TCs can induce M2 TAMs with increased expression of PD-L1 $(38,39)$. It is also known that PD-L1, induced by IFN- $\gamma$ from TAMs, promoted the progression of lung cancer (40). Recent studies show that other signals derived from macrophages, such as TNF- $\alpha$, VEGF and CXCL8, can induce PD-L1 expression (41-43). In addition, previous studies report that macrophages can induce not only PD-L1 expression, but also PD-L2 expression $(44,45)$.

From these findings, the TIL and M2 TAM densities were associated with the expression of PD-L1 and PD-L2 on TCs and ICs. In the present study, the TIL density was significantly associated with the preoperative serum albumin level $(r=0.269$; $\mathrm{P}<0.001$; Fig. S1A) and the preoperative peripheral blood lymphocyte count $(r=0.209 ; \mathrm{P}=0.006$; Fig. S1B). Therefore, TILs are considered to be a host-related factor. By contrast, M2 TAMs are considered to be a tumor-related factor (23). Thus, such complex crosstalk in the TME, including TILs and M2 TAMs, could affect the expression of PD-L1 and PD-L2 on TCs and ICs in NSCLC (46).

However, the present study demonstrated the additional finding of no correlation between PD-L1 expression on TCs and PD-L2 expression on TCs, despite the possible same regulations by TILs and M2 TAMs. Several studies also report a high frequency of discordance between PD-L1 and PD-L2 expression in human cancer $(47,48)$. By contrast, there were correlations between PD-L1 expression on TCs and PD-L1 expression on ICs and between PD-L2 expression on TCs and PD-L2 expression on ICs in the present study.

The present study revealed that tumor differentiation was strongly associated with PD-L1 expression on TCs and ICs. The percentages of PD-L1-positive TCs and PD-L1-positive ICs were higher in poorly differentiated tumors compared with that in well- and moderately differentiated tumors. A meta-analysis on PD-L1 expression in lung cancer also reports the same results (49). In addition, an experimental study reveals that $\mathrm{PD}-\mathrm{L} 1$ could upregulate the $\beta$-catenin signaling pathway to induce epithelial-mesenchymal transition (50), which is associated with tumor differentiation in lung cancer $(51,52)$. By contrast, tumor differentiation was inversely associated with PD-L2 expression on TCs and ICs in the present study. The percentages of PD-L2-positive TCs and PD-L2-positive ICs were higher in well-differentiated tumors compared with that in poorly and moderately differentiated tumors.

Therefore, the combined evaluation of PD-L1 and PD-L2 expression could be considered clinically important in the treatment strategy of immune-checkpoint inhibitors in patients with NSCLC. In particular, the evaluation of PD-L2 expression may be necessary for patients with PD-L1-negative NSCLC. Patients with PD-L2-positive NSCLC could be treated with anti-PD-1 antibodies, such as Pembrolizumab, and combined treatment with anti-PD-L2 antibodies in the future (18-20). In fact, in the present study, immune-checkpoint inhibitors were only used in 7 cases of PD-L1-positive tumors at the time of disease recurrence, whereas 56 cases had recurrence following surgery. Further clinical studies are required for patients with PD-L2-positive NSCLC. In addition, the present study was performed using a relatively small number of patients at one institution. Therefore, a further study using more cases is required to elucidate the clinical significance of PD-L2 expression, especially with respect to the treatment strategy of immune-checkpoint inhibitors. Furthermore, the present study was evaluated only by immunohistochemistry and a further study to investigate their gene copy numbers may be needed (53).

In conclusion, PD-L1 and PD-L2 expression on TCs and ICs was associated with TILs and M2 TAMs in NSCLC. However, there was no correlation between PD-L1 and PD-L2 expression on TCs. Meanwhile, PD-L1 expression on TCs and ICs was associated with tumor differentiation, while PD-L2 expression on TCs and ICs was inversely associated with tumor differentiation. The combined evaluation of PD-L1 and PD-L2 expression could be considered clinically important in the treatment strategy of immune-checkpoint inhibitors in patients with NSCLC. In particular, the evaluation of PD-L2 expression may be necessary for patients with PD-L1-negative NSCLC.

\section{Acknowledgements}

Not applicable.

\section{Funding}

No funding was received.

\section{Availability of data and materials}

The datasets used and/or analyzed during the current study are available from the corresponding author on reasonable request.

\section{Authors' contributions}

RS, CLH and HD designed the study. RS, CLH and MF designed and performed the experiments. RS, CLH and HC collected the data. RS and CLH analyzed and interpreted the data and wrote the manuscript. RS and CLH confirm the authenticity of all the raw data. All authors have read and approved the final version of the manuscript for publication.

\section{Ethics approval and consent to participate}

The current study was approved by the Institutional Ethics Committee of the Kitano Hospital (approval no. P181200300) and written informed consent was provided from each patient. The research was conducted in compliance with the principles outlined in the Declaration of Helsinki.

\section{Patient consent for publication}

Written informed consent for publication of patient data/accompanying images was obtained.

\section{Competing interests}

The authors declare that they have no competing interests. 


\section{References}

1. Ettinger DS, Akerley W, Bepler G, Blum MG, Chang A, Cheney RT, Chirieac LR, D'Amico TA, Demmy TL, Ganti AK, et al: Non-small cell lung cancer. J Natl Compr Canc Netw 8: 740-801, 2010

2. Hsu WH, Yang JC, Mok TS and Loong HH: Overview of current systemic management of EGFR-mutant NSCLC. Ann Oncol 29 (Suppl_1): i3-i9, 2018.

3. Borghaei H, Paz-Ares L, Horn L, Spigel DR, Steins M, Ready NE, Chow LQ, Vokes EE, Felip E, Holgado E, et al: Nivolumab versus docetaxel in advanced nonsquamous non-small-cell lung cancer. N Engl J Med 373: 1627-1639, 2015.

4. Herbst RS, Baas P, Kim DW, Felip E, Perez-Gracia JL and Han JY: Pembrolizumab versus docetaxel for previously treated, PD-L1-positive, advanced non-small-cell lung cancer (KEYNOTE-010): A randomized controlled trial. Lancet 387: $1540-1550,2016$.

5. Rittmeyer A, Barlesi F, Waterkamp D, Park K, Ciardiello F, von Pawel J, Gadgeel SM, Hida T and Kowalski DM: Atezolizumab versus docetaxel in patients with previously treated non-small-cell lung cancer (OAK): A phase 3, open-label, multicenter randomized controlled trial. Lancet 389: 255-265, 2017.

6. Kowanetz M, Zou W, Gettinger SN, Koeppen H, Kockx M, Schmid P, Kadel EE, Wistuba I, Chaft J, Rizvi NA, et al: Differential regulation of PD-L1 expression by immune and tumor cells in NSCLC and the response to treatment with atezolizumab (anti-PD-L1). Proc Natl Acad Sci USA 115 E10119-E10126, 2018.

7. Kim MY, Koh J, Kim S, Go H, Jeon YK and Chung DH: Clinicopathological analysis of PD-L1 and PD-L2 expression in pulmonary squamous cell carcinoma: Comparison with tumor-infiltrating $\mathrm{T}$ cells and the status of oncogenic drivers. Lung Cancer 88: 24-33, 2015.

8. Sumitomo R, Hirai T, Fujita M, Murakami H, Otake Y and Huang C: PD-L1 expression on tumor-infiltrating immune cells is highly associated with M2 TAM and aggressive malignant potential in patients with resected non-small cell lung cancer. Lung Cancer 136: 136-144, 2019.

9. Shinchi Y, Komohara Y, Yonemitsu K, Sato K, Ohnishi K, Saito Y, Fujiwara Y, Mori T, Shiraishi K, Ikeda K and Suzuki M: Accurate expression of PD-L1/L2 in lung adenocarcinoma cells: A retrospective study by double immunohistochemistry. Cancer Sci 110: 2711-2721, 2019

10. Matsubara T, Takada K, Azuma K, Takamori S, Toyokawa G, Haro A, Osoegawa A, Tagawa T, Kawahara A, Akiba J, et al: A clinicopathological and prognostic analysis of PD-L2 expression in surgically resected primary lung squamous cell carcinoma. Ann Surg Oncol 26: 1925-1933, 2019.

11. Takamori S, Takada K, Azuma K, Jogo T, Shimokawa M, Toyokawa G, Hirai F, Tagawa T, Kawahara A, Akiba J, et al: Prognostic impact of programmed death-ligand 2 expression in primary lung adenocarcinoma patients. Ann Surg Oncol 26: 1916-1924, 2019.

12. Baptista MZ, Sarian LO, Derchain SFM, Pinto GA and Vassallo J: Prognostic significance of PD-L1 and PD-L2 in breast cancer. Hum Pathol 47: 78-84, 2016.

13. Zhao SG, Lehrer J, Chang SL, Das R, Erho N, Liu Y, Sjostrom M, Den RB, Freedland SJ, Klein EA, et al: The immune landscape of prostate cancer and nomination of PD-L 2 as a potential therapeutic target. J Natl Cancer Inst 111: 301-310, 2019.

14. Okadome K, Baba Y, Nomoto D, Yagi T, Kalikawe R, Harada K, Hiyoshi Y, Nagai Y, Ishimoto T, Iwatsuki M, et al: Prognostic and clinical impact of PD-L2 and PD-L1 expression in a cohort of 437 oesophageal cancers. Br J Cancer 122: 1535-1543, 2020.

15. Okazaki T and Honjo T: PD-1 and PD-1 ligands: From discovery to clinical application. Int Immunol 19: 813-824, 2007.

16. Rozali EN, Hato SV, Robinson BW, Lake RA and Lesterhuis WJ: Programmed death ligand 2 in cancer-induced immune suppression. Clin Dev Immunol 2021: 656340, 2012.

17. Zhong X, Tumang JR, Gao W, Bai C and Rothstein TL: PD-L2 expression extends beyond dendritic cells/macrophages to $\mathrm{B} 1$ cells enriched for $\mathrm{V}(\mathrm{H}) 11 / \mathrm{V}(\mathrm{H}) 12$ and phosphatidylcholine binding. Eur J Immunol 37: 2405-2410, 2007.

18. Tanegashima $\mathrm{T}$, Togashi $\mathrm{Y}$, Azuma K, Kawahara A, Ikeguchi K, Sugiyama D, Kinoshita F, Akiba J, Kashiwagi E, Takeuchi A, et al: Immune suppression by PD-L2 against spontaneous and treatment-related antitumor immunity. Clin Cancer Res 25: 4808-4819, 2019.
19. Umezu D, Okada N, Sakoda Y, Adachi K, Ojima T, Yamaue H, Eto $\mathrm{M}$ and Tamada K: Inhibitory functions of PD-L1 and PD-L2 in the regulation of anti-tumor immunity in murine tumor microenvironment. Cancer Immunol Immunother 68: 201-211, 2019.

20. Yearley JH, Gibson C, Yu N, Moon C, Murphy E, Juco J, Lunceford J, Cheng J, Chow LQM, Seiwert TY, et al: PD-L2 Expression in Human Tumors: Relevance to Anti-PD-1 Therapy in Cancer. Clin Cancer Res 23: 3158-3167, 2017.

21. Amin MB, Edge S and Greene F: AJCC Cancer Staging Manual 8th edition. Springer, New York, 2017.

22. Travis WD, Brambilla E, Burke AP, Marx A and Nicholson AG: WHO Classification of Tumours of the Lung, Pleura, Thymus and Heart. 4th edition. International Agency for Research on Cancer, Lyon, France, 2015.

23. Sumitomo R, Hirai T,Fujita M, Murakami H,Otake Y and Huang C: M2 tumor-associated macrophages promote tumor progression in non-small-cell lung cancer. Exp Ther Med 18: 4490-4498, 2019.

24. Tsao MS, Kerr KM, Kockx M, Beasley M, Borczuk AC, Botling J, Budendorf L, Chirieac L, Chen G, Chou T, et al: PD-L1 immunohistochemistry comparability study in real-life clinical samples: Results of Blueprint print phase 2 project. J Thorac Oncol 13: 1302-1311, 2018.

25. Li Z, Maeda D, Yoshida M, Umakoshi M, Nanjo H, Shiraishi K, Saito M, Kohno T, Konno H, Saito H, et al: The intratumoral distribution influences the prognostic impact of CD68- and CD204-positive macrophages in non-small cell lung cancer. Lung Cancer 123: 127-135, 2018.

26. Tanaka R, Ichimura Y, Kubota N, Saito A, Nakamura Y, Ishitsuka Y, Watanabe R, Fujisawa Y, Mizuno S, Takahashi S, et al: Differential involvement of programmed cell death ligands in skin immune responses. J Invest Dermatol 142: 145-154.e8, 2022.

27. Sanmamed MF and Chen L: Inducible expression of B7-H1 (PD-L1) and its selective role in tumor site immune modulation. Cancer J 20: 256-261, 2014.

28. Chen J, Feng Y, Lu L, Wang H, Dai L, Li Y and Zhang P: Interferon- $\gamma$-induced PD-L1 surface expression on human oral squamous carcinoma via PKD2 signal pathway. Immunobiology 217: 385-393, 2012.

29. Lane RS, Femel J, Breazeale AP, Loo CP, Thibault G, Kaempf A, Mori M, Tsujikawa T, Chang YH and Lund AW: IFN $\gamma$-activated dermal lymphatic vessels inhibit cytotoxic $\mathrm{T}$ cells in melanoma and inflamed skin. J Exp Med 215: 3057-3074, 2018.

30. Qian J, Wang C, Wang B, Yang J, Wang Y, Luo F, Xu J, Zhao C, Liu R and Chu Y: The IFN- $\gamma / P D-L 1$ axis between T cells and tumor microenvironment: Hints for glioma anti-PD-1/PD-L1 therapy. J Neuroinflammation 15: 290, 2018.

31. Chen S, Crabill GA, Pritchard TS, McMiller TL, Wei P, Pardoll DM, Pan F and Topalian SL: Mechanisms regulating PD-L1 expression on tumor and immune cells. J Immunother Cancer 7: 305, 2019.

32. Arrieta O, Montes-Servin E, Hernandez-Martinez J, Cardona AF, Cases-Ruiz E, Crispin JC, Motola D, Flores-Estrada D and Barrera L: Expression of PD-1/PD-L1 and PD-L2 in peripheral T-cells from non-small cell lung cancer patients. Oncotarget 8: 101994-102005, 2017.

33. Kitsou M, Ayiomamitis GD and Zaravinos A: High expression of immune checkpoints is associated with the TIL load, mutation rate and patient survival in colorectal cancer. Int J Oncol 57: 237-248, 2020.

34. Zhang Y, Xu J, Hua J, Liu J, Liang C, Meng Q, Wei M, Zhang B and $\mathrm{Yu}$ X: A PD-L2-based immune marker signature helps to predict survival in resected pancreatic ductal adenocarcinoma. J Immunotherapy Cancer 7: 233, 2019.

35. Mei J, Xiao Z, Guo C, Pu Q, Ma L, Liu C, Lin F, Liao H, You Z and Liu L: Prognostic impact of tumor-associated macrophage infiltration in non-small cell lung cancer: A systemic review and meta-analysis. Oncotarget 7: 34217-34228, 2016.

36. Jackute J, Zemaitis M, Pranys D, Sitkauskiene B, Miliauskas S, Vaitkiene S and Sakalaukas R: Distribution of M1 and M2 macrophages in tumor islets and stroma in relation to prognosis of non-small cell lung cancer. BMC Immunol 19: 3, 2018.

37. Mantovani A, Sozzani S, Locati M, Allavena P and Sica A: Macrophage polarization: Tumor-associated macrophages as a paradigm for polarized M2 mononuclear phagocytes. Trends Immunol 23: 549-555, 2002.

38. Gabrusiewicz K, Li X, Wei J, Hashimoto Y, Marisetty AL, Ott M, Wang F, Hawke D, Yu J, Healy LM, et al: Glioblastoma stem cell-derived exosomes induce M2 macrophages and PD-L1 expression on human monocytes. Oncoimmunology 7: e1412909, 2018. 
39. Wen ZF, Liu H, Gao R, Zhou M, Ma J, Zhang Y, Zhao J, Chen Y, Zhang T, Huang F, et al: Tumor cell-released autophagosomes (TRAPs) promote immunosuppression through induction of M2-like macrophages with increased expression of PD-L1. J Immunother Cancer 6: 151, 2018.

40. Zhang X, Zeng Y, Qu Q, Zhu J, Liu Z, Ning W, Zeng H, Zhang N, $\mathrm{Du}$ W, Chen C and Huang JA: PD-L1 induced by IFN- $\gamma$ from tumor-associated macrophages via the JAK/STAT3 and $\mathrm{PI} 3 \mathrm{~K} / \mathrm{AKT}$ signalling pathways promoted progression of lung cancer. Int J Clin Oncol 22: 1026-1033, 2017.

41. Tsukamoto $M$, Imai $K$, Ishimoto $T$, Komohara $Y$, Yamashita Y, Nakagawa S, Umezaki N, Yamao T, Kitano Y, Miyata T, et al: PD-L1 expression enhancement by infiltrating macrophage-derived tumor necrosis factor- $\alpha$ leads to poor pancreatic cancer prognosis. Cancer Sci 110: 310-320, 2019.

42. Lai YS, Wahyuningtyas R, Aui SP and Chang KT: Autocrine VEGF signalling on M2 macrophages regulates PD-L1 expression for immunomodulation of T cells. J Cell Mol Med 23: $1257-1267,2019$.

43. Lin C, He H, Liu H, Li R, Chen Y, Qi Y, Jiang Q, Chen L, Zhang P, Zhang H, et al: Tumor-associated macrophage-derived CXCL8 determines immune evasion through autonomous PD-L1 expression in gastric cancer. Gut 68: 1764-1773, 2019.

44. Horlad H, Ma C, Yano H, Pan C, Ohnishi K, Fujiwara Y, Endo S, Kikukawa Y, Okuno Y, Matsuoka M, et al: An IL-27/Stat3 axis induces expression of programmed death ligands (PD-L1/2) on infiltrating macrophages in lymphoma. Cancer Sci 107: 1696-1704, 2016

45. Cai X, Yuan F, Zhu J, Yang J, Tang C, Cong Z and Ma C: Glioma-associated stromal cells stimulate glioma malignancy by regulating the tumor immune microenvironment. Front Oncol 11: 672928, 2021.

46. Wang J, Li D, Cang H and Guo B: Crosstalk between cancer and immune cells: Role of tumor-associated macrophages in the tumor microenvironment. Cancer Med 8: 4709-4721, 2019.
47. Menguy S, Prochazkova-Carlotti M, Beylot-Barry M, Saltel F, Vergier B, Merlio J and Pham-Ledard A: PD-L1 and PD-L2 are differentially expressed by macrophages or tumor cells in primary cutaneous diffuse large B-cell lymphoma, Leg type. Am J Surg Pathol 42: 326-334, 2018.

48. Pinato DJ, Vallipuram A, Evans JS, Wong C, Zhang $\mathrm{H}$, Brown M, Dina RE, Trivedi P, Akarca AU, Marafioti T, et al: Programmed cell death ligand expression drives immune tolerogenesis across the diverse subtypes of neuroendocrine tumors. Neuroendocrinology 111: 465-474, 2021.

49. Li H, Xu Y, Wan B, Song Y, Zhan P, Hu Y, Zhang Q, Zhang F, Liu H, Li T, et al: The clinicopathological and prognostic significance of PD-L1 expression assessed by immunohistochemistry in lung cancer: A meta-analysis of 50 studies with 11,383 patients. Transl Ling Cancer Res 8: 429-449, 2019.

50. Yu W, Hua Y, Qiu H, Hao J, Zou Z, Li Z, Hu S, Guo P, Chen M, Sui S, et al: PD-L1 promotes tumor growth and progression by activating WIP and $\beta$-catenin signaling pathways and predicts poor prognosis in lung cancer. Cell Death Dis 11: 506, 2020.

51. Sato M, Shames DS and Hasegawa Y: Emerging evidence of epithelial-to-mesenchymal transition in lung carcinogenesis. Respirology 17: 1048-1059, 2012.

52. Brabletz S, Schuhwerk H, Brabletz T and Stemmler MP: Dynamic EMT: A multi-tool for tumor progression. EMBO J 40: e108647, 2021.

53. Inoue $\mathrm{Y}$, Yoshimura $\mathrm{K}$, Nishimoto $\mathrm{K}$, Inui $\mathrm{N}$, Karayama $\mathrm{M}$, Yasui H, Hozumi H, Suzuki Y, Furuhashi K, Fujisawa T, et al: Evaluation of programmed death ligand 1 (PD-L1) gene amplification and response to nivolumab monotherapy in non-small cell lung cancer. JAMA Netw Open 3: e2011818, 2020.

(i) $(5)$ This work is licensed under a Creative Commons Attribution-NonCommercial-NoDerivatives 4.0 International (CC BY-NC-ND 4.0) License. 